\title{
Distributed MPC of wide-area electromechanical oscillations of large-scale power systems
}

\author{
D. Wang M. Glavic L. Wehenkel \\ Department of Electrical Engineering and Computer Science \\ University of Liège Sart-Tilman, B28 - Liège B4000, Belgium \\ Da.Wang@student.ulg.ac.be, glavic@montefiore.ulg.ac.be, L.Wehenkel@ulg.ac.be
}

\begin{abstract}
We investigate distributed Model Predictive Control (MPC) to damp wide-area electromechanical oscillations. Our distributed MPC schemes are derived from and compared with a fully centralized MPC scheme proposed in a previous publication. Based on simulations carried out using a 16-generator, 70-bus, two-area test power system, we show that simple coordination schemes based on additional local measurements' feedback yield already a significant improvement with respect to a scheme with only implicit coordination, improve significantly with respect to purely local controls, and in this respect reach about $75 \%$ of the improvements obtained by an ideal centralized MPC scheme.

Index Terms-distributed MPC, inter-area oscillations, decomposition, coordination.
\end{abstract}

\section{INTRODUCTION}

Wide-area low-frequency electromechanical oscillations more and more often threaten the secure operation of modern power systems [1]-[3]. Indeed, the characteristics of modern power systems, such as long transmission distances over weak grids, highly variable generation patterns and heavy loading, tend to increase the probability of appearance of sustained wide-area electromechanical oscillations likely to lead to large-scale blackouts [2], [3]. While these oscillations are slow enough to envisage wide-area control approaches, their dynamics are nevertheless beyond the capabilities of human operator based control. It is therefore necessary to design automatic control schemes to address them.

In this context, current control systems are based on very local control strategies (e.g., Power System Stabilizers (PSSs) using "frozen" feedback loops designed in off-line studies). To overcome the limitations of these systems, a promising option would be to automatically adjust their control strategy to the changing behavior of the underlying system (caused, e.g., by outages of control devices, maintenance operations, connectivity changes, incorporation of new generation, modifications of load dynamics implied by smart-grid technologies, etc.). Since power system dynamics can be quite accurately modeled, and given the recent progresses in large-scale optimization, a natural idea is to apply Model Predictive Control (MPC) to design such adaptive control strategies [4].

In the process control industry, MPC is considered as a proven technique with numerous real-life applications [5]. The principle of MPC can be shortly summarized as follows. At any time, the MPC algorithm uses the collected measurements and a model of the system and a specification of the control objective, in order to compute an optimal open-loop control sequence over a specified time horizon. The first-stage controls computed in this way are applied to the system, and the entire procedure is repeated at subsequent steps as soon as measurements (or model) updates become available [6].

However, since wide-area power system oscillations tend to appear in very large-scale systems, ranging over thousands of kilometers and involving many different subsystems managed by different transmission system operators (TSOs), it is often practically not feasible to handle these problems with a fully centralized approach. On the other hand, reliability/vulnerability considerations may suggest that even in a system where a fully centralized control scheme would be feasible, it is not necessarily desirable to do so. Consequently, it is of interest to study distributed MPC schemes addressing various decompositions of the global control problem. In this setting, local MPC systems could determine optimal inputs for a subset of controllers under their authority, based on a model of their subsystem and a local control objective [7], [8].

Security constrained optimal power flow [9] and coordinated secondary voltage control [10] pioneered MPC in power systems. More recently MPC was proposed for emergency alleviation of thermal overloads [11]-[13], voltage control [14][16], transient stability and oscillations control [4], [17], [18], automatic generation control [19], [20]. In [12], agents are placed at each generator and load to control injections, and they use detailed models of their surrounding area and simplified models of remote ones; they share their objectives and exchange solutions and measurements. By cloning the boundary nodes of neighboring areas, reference [15] breaks the whole power grid into relatively independent subsystems that only interact through consistency constraints on shared variables; each local MPC controller calculates optimal inputs for automatic voltage regulators and static Var compensators in its area, and coordinates with neighboring MPC controllers by exchanging Lagrange multipliers.

In this paper we consider distributed MPC for damping wide-area electromechanical oscillations. The paper is organized as follows. Section II recalls the centralized MPC problem formulation from [4], while Section III proposes a distributed MPC scheme and discusses its decomposition and coordination issues. In Section IV, three distributed schemes, two of them involving weak coordination by Wide Area Measurement Systems (WAMS), are proposed and assessed. Section V highlights conclusions and research directions. 


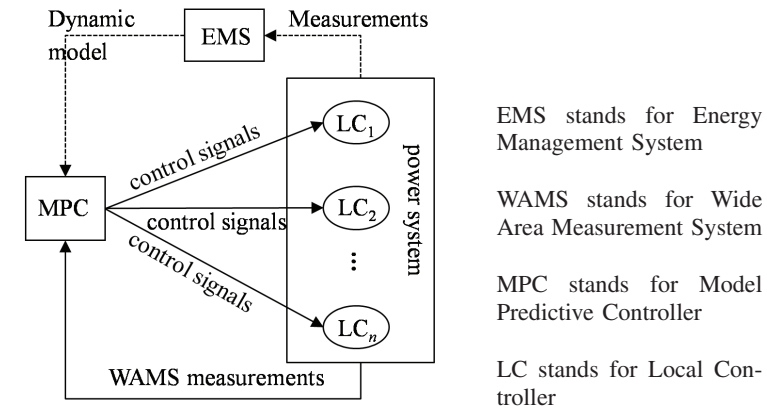

Fig. 1. Schematic diagram of the centralized MPC scheme [4].

\section{Centralized MPC SCHEME FOR Wide-AREA ELECTROMECHANICAL OSCILLATIONS DAMPING [4]}

For the sake of making this paper self-contained and to introduce the notations and concepts used subsequently, we briefly recall in this section the centralized MPC scheme proposed in [4]. This scheme is based on a linearized discrete-time model of the power system dynamics combined with a linearly constrained quadratic optimal control problem formulation.

\section{A. Discrete time linearized dynamic system model}

The MPC formulation of [4] (see Figure 1) is based on a state-space model of a multi-machine power system in the form of the following linearized continuous time model:

$$
\left\{\begin{array}{l}
\dot{x}=A_{c} x+B_{c} u \\
y=C_{c} x
\end{array}\right.
$$

where $x \in R^{m_{x}}$ is a vector of state variables, $u \in R^{m_{u}}$ is a vector of controls (inputs), and $y \in R^{m_{y}}$ is a vector of performance measurements (outputs).

Next, from Eqn (1) the transition for a small step of $\delta$ seconds is inferred by:

$$
\left\{\begin{array}{l}
x(t+\delta)=\left(\delta A_{c}+I\right) x(t)+\delta B u(t) \\
y(t)=C x(t)
\end{array}\right.
$$

yielding a discrete-time dynamics (for time steps $k \delta$ ) given by

$$
\begin{aligned}
& x[k+1]=A x[k]+B u[k] ; y[k]=C x[k], \\
& \text { with: } A=\left(\delta A_{c}+I\right) ; B=\delta B_{c} ; C=C_{c} .
\end{aligned}
$$

\section{B. MPC formulation as a quadratic optimization problem}

The MPC problem is formulated as an optimization problem over $K$ time steps. At time $t$, based on the estimation $\hat{x}(t)$ of the current system state (e.g., obtained from a state estimator fed from a WAMS) and on the system model, the predicted outputs $\hat{y}(t+k \delta)=\hat{y}[k]$ over the next horizon are obtained by iterating Eqn (3) $K$ times by using $\hat{x}(t)$ as initial state:

$$
\left[\begin{array}{c}
\hat{y}[1] \\
\hat{y}[2] \\
\vdots \\
\hat{y}[K]
\end{array}\right]=P_{x} \hat{x}(t)+P_{u}\left[\begin{array}{c}
u[0] \\
u[1] \\
\vdots \\
u[K-1]
\end{array}\right]
$$

where $P_{x}$ and $P_{u}$ are given by

$$
P_{x}=\left[\begin{array}{c}
C A \\
C A^{2} \\
\vdots \\
C A^{K}
\end{array}\right], P_{u}=\left[\begin{array}{cccc}
C B & 0 & \ldots & 0 \\
C A B & C B & \ldots & 0 \\
\vdots & \vdots & \ddots & \vdots \\
C A^{K-1} B & C A^{K-2} B & \ldots & C B
\end{array}\right] .
$$

Using these equations, the following quadratic optimization problem is solved at every time step:

$$
\min _{u[\cdot]}\left\{\sum_{i=1}^{K}\left(\hat{y}[i]-y_{r}\right)^{T} Q\left(\hat{y}[i]-y_{r}\right)\right\}
$$

subject to linear inequality constraints $(\forall i=0, \ldots, K-1)$ :

$$
\begin{aligned}
& u_{\min } \leq u[i] \leq u_{\max } \\
& z_{\min } \leq z[i+1] \leq z_{\max }
\end{aligned}
$$

where $Q$ is a symmetric positive definite matrix of weight factors, $y_{r}$ is the vector of performance targets, and $z$ is a vector of constrained variables (like currents or voltages).

More details about this MPC model and algorithm are provided in reference [4] .

\section{Distributed MPC SCHEME FOR WIDE-AREA ELECTROMECHANICAL OSCILLATIONS DAMPING}

In this section we consider the extension of the above formulation towards a distributed MPC implementation, which would be a more viable solution for damping low-frequency oscillations in very large-scale interconnected power systems. As with any other distributed control scheme, two problems are to be solved, namely decomposition and coordination.

\section{A. Control problem decomposition}

A large-scale control problem can be decomposed into subproblems by the following two main approaches:

- Problem-driven: construction of a global system model followed by an "optimal" decomposition into subsystems according to structural properties of the system and the control problem under consideration [21],

- Context-driven: the decomposition of the whole system is imposed by contingent constraints, and hence the construction of the local control schemes has to follow the already given decomposition [15], [16].

Considering information-exchange restrictions in certain power grids, and organizational barriers, it is quite difficult to construct an exact system-wide model, and even if this were possible, it would be difficult to impose significant changes within the existing control structures unless they would keep aligned with existing TSO responsibility areas. We therefore prefer to consider in this paper a context-driven decomposition of control areas [20], although we believe that our approaches could as well be used in order to readjust the boundaries of control areas for the sake of overall system performance, but these considerations are beyond the subject of this paper.

Let us consider a two-area system, as in Fig. 2, to illustrate our approach. Notice that this system is decomposed a priori into two control areas, linked by tie lines allowing to exchange 


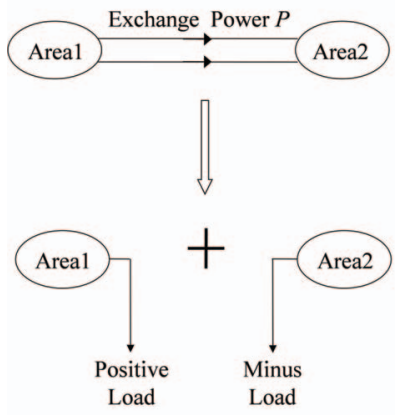

Fig. 2. Decomposition of an interconnected grid into two subsystems (top); possible view of the whole system by each subsystem (bottom)

power. A control system only able to act on one of the two subsystems could view the rest of the system by two equivalent dynamic loads in order to compute its control inputs. Accordingly, we rewrite the global control objective of Eqn. (5) as the simultaneous and parallel resolution of the following area-wise problems (subscript $n$ refers to area $n$ ):

$$
\min _{u_{n}[\cdot]}\left\{\sum_{i=1}^{K_{n}}\left(\hat{y}_{n}[i]-y_{r . n}\right)^{T} Q_{n}\left(\hat{y}_{n}[i]-y_{r . n}\right)\right\}
$$

subject to linear inequality constraints $(\forall i=0, \ldots, K-1)$ :

$$
\begin{aligned}
& u_{\min . n} \leq u_{n}[i] \leq u_{\max . n} \\
& z_{\min . n} \leq z_{n}[i+1] \leq z_{\max . n}
\end{aligned}
$$

In this scheme, each local MPC controller solves its optimization problem using a detailed model of its own area and a possibly very rough model of the remaining areas (typically a black box model). It then sends the first inputs in the computed optimal control sequence to the controllers under its responsibility, and observes the resulting effects to proceed.

\section{B. Coordination of controls of subsystems}

Since distributed MPC controllers act in the same system, coordination is needed in order to achieve satisfactory performances in damping low-frequency oscillations throughout the system [8], [12], [19]. They could negotiate/exchange useful information in order to improve global performance. E.g., local agents of [12] are designed to inform their neighbors about what they intend to do and pass along measurements that other agents may not be able to sense directly. In [20], local agents exchange Lagrange multipliers and values of shared variables after each computation and computations are carried on until absolute changes in the Lagrange multipliers are smaller than a pre-defined small positive constant, before actual controls are applied to the system.

For the damping of wide-area electromechanical power system oscillations, dynamic behaviors evolve continuously and possibly in a rather unpredictable way. Since the controls needed to stabilize these dynamic phenomena may pertain to very remote subsystems, thousands of kilometers away from each other and separated by many intermediate areas, we will investigate weaker coordination techniques, which we believe

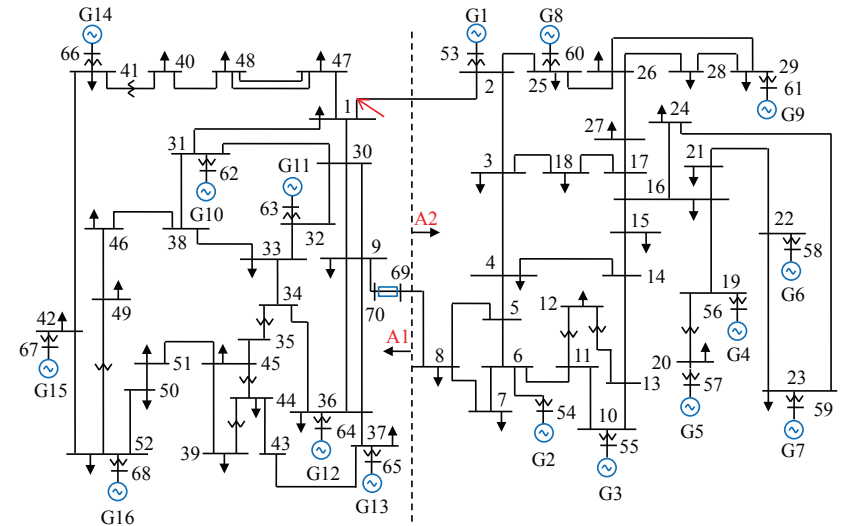

Fig. 3. 16 generators / 70 bus system

to be preferable in this context. Specifically, we will first study an implicit coordination scheme [7], where in the spirit of primary frequency control, each area tries to solve its own problem and where the overall system stability is hoped to emerge from these area-wise controls. After the analysis of this implicit coordination scheme, we propose two weak explicit coordination schemes in order to improve performances.

\section{Distributed MPC CONTROL SChemes}

\section{A. Illustrative problem used for empirical evaluations}

In order to illustrate the proposed distributed MPC schemes, we use the system of Figure 3. A TCSC is installed between buses 69 and 70 and there is a PSS on each generator. The system contains two areas: A1 and A2. The TCSC is assigned to area $\mathrm{A} 1$ as its control resource. In our simulations, we replace the real-system by a non-linear time domain simulation software from the MATLAB Power System Toolbox (PST) [1] and use $K=300$ corresponding to $K \delta=\Delta T=1.5 \mathrm{~s}$ ). All models come from the PST software.

In our test scenarios, a temporary three-phase short-circuit to ground at bus 1 causes oscillations between A1 and A2. Figure 4 shows respectively the temporal evolution over a period of 30s of the power flow through line 1-2 and the angular speeds of all generators, when no MPC controller is used. We observe sustained, slowly damped oscillations.

In all our simulations, MPC1 is responsible for PSS1016 and the TCSC; MPC2 calculates inputs for PSS1-9. The state vectors of the two MPC controllers contain generator, exciter, PSS and turbine governor states of the elements in their control area (MPC1's state vector also contains 1 TCSC state). Both output vectors $y_{i}$ compile the generator speeds for each area, whose references $y_{r}$ are the unit vectors of dimension 7 and 9 respectively. Input vector $u_{1}$ of MPC1 consists of supplementary inputs for PSS10-16 and TCSC, while MPC2 inputs $\left(u_{2}\right)$ are supplementary inputs for PSS1-9. All inputs are subject to $-0.1 \leq u \leq 0.1$. Because of limitations in the PST model implementations, we use equivalent supplementary inputs to exciters to substitute for those supplementary inputs to PSSs. We use in our simulations a prediction horizon of 

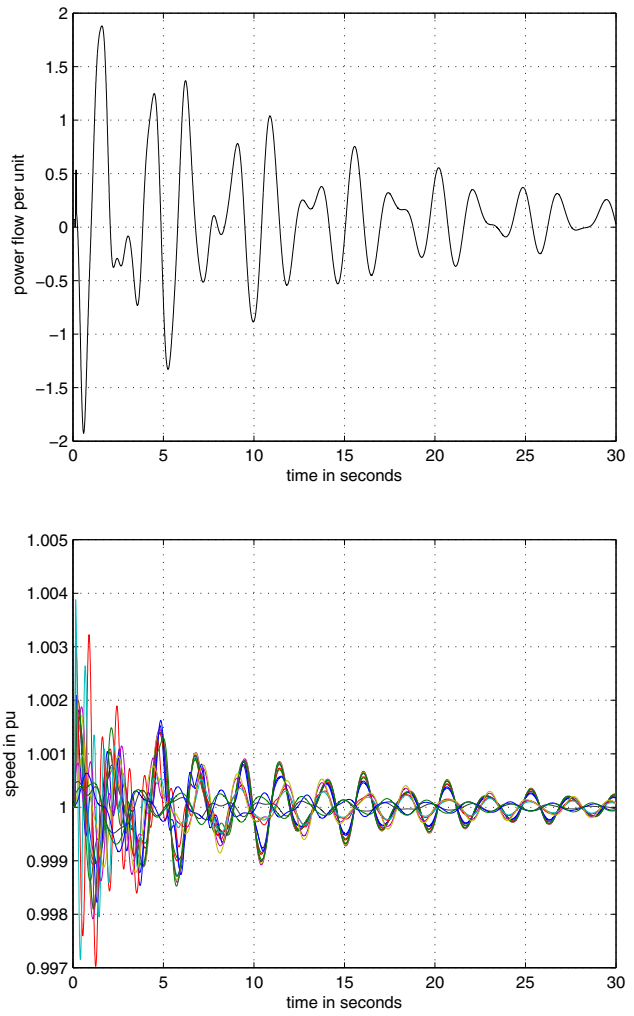

Fig. 4. Response without MPC controller: (top) active power of tie-line 1-2; (bottom) angular speeds

$H_{p}=15$ discrete steps of $\Delta t=0.1$ s (i.e. a prediction horizon of 1.5s) and a control horizon $H_{u}=3$ (i.e. the input is changed over the next $H_{u}$ steps), as in [4]. To assess the power system response, we simulate and observe it over a period of $20 \mathrm{~s}$ or 30s. In the objective function (7), all deviations of the predicted outputs from references are weighted uniformly and independently, i.e. $Q_{n}$ is the identity matrix.

\section{B. Implicit coordination of MPC controllers}

In this setting, we use independent MPC controllers for each area which use only local measurements and essentially ignore the rest of the system.

Controlled system responses over a time period of 20 s of the power flow through line1-2 and the angular speed of a representative generator are shown by solid curves in Fig 5 . Compared with the system response without MPC, namely dashed curves in Fig 5, we clearly observe that this (trivially) distributed MPC scheme effectively damps the power oscillations of the tie-line and the angular speed of generator 1.

Compared with the fully centralized MPC scheme (dashed curves of Fig 6, from [4]), control effects of this first distributed MPC scheme are still quite inferior. This is not so astonishing, because the formulation of the optimal solutions of the local MPC schemes do not provide any guarantee in terms of global optimal solution optimality. (Paper [8] discusses conditions under which optimal solutions of sub-
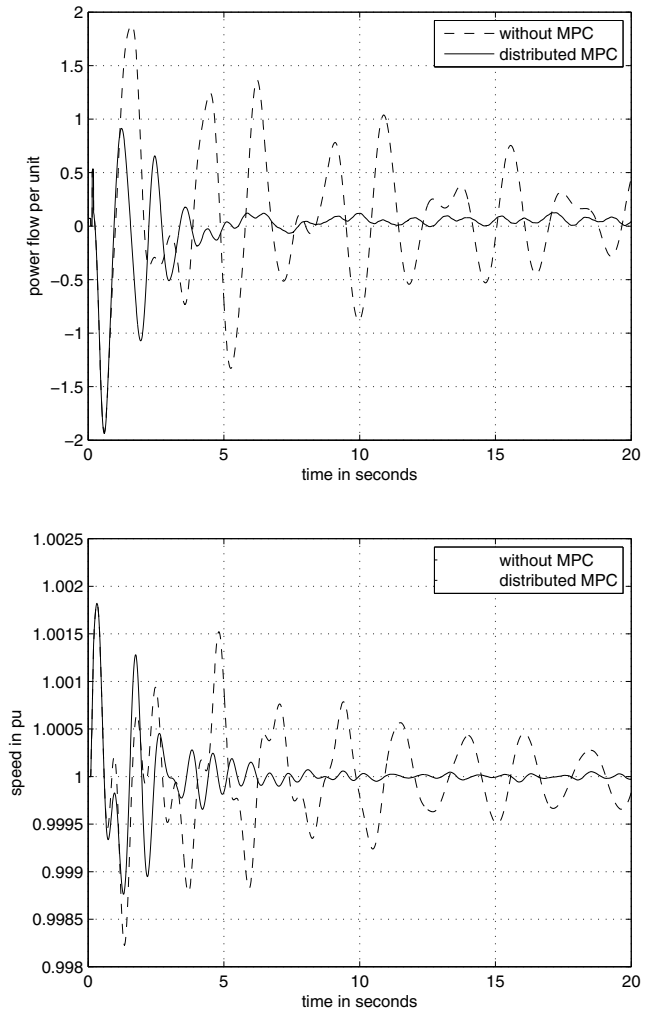

Fig. 5. Response without MPC (dashed) and with distributed MPC (plain): (top) active power of tie-line 1-2; (bottom) angular speed of generator 1

problems compose an optimal solution of the overall problem. But they unfortunately do not apply to the present scheme.)

We also note that although this trivial distributed MPC scheme quickly damps large oscillations within the first $5 \mathrm{~s}$, small oscillations remain and last from $5 \mathrm{~s}$ to $20 \mathrm{~s}$. These small oscillations are possibly caused by the inaccurate and incomplete models used by local MPC controllers.

\section{Weakly coordinated MPC scheme I}

Each MPC based controller of the above considered scheme does not account for what is happening in other system areas, and does not consider possible interactions with its neighbors when it computes its control decisions. But actual angular speeds in one area depend not only on inputs of its own MPC controller, but also on the response of the whole system. This is why the combination of area-wise MPC controllers is not able to make angular speeds reach reference values and why small oscillations permanently remain in the above uncoordinated distributed MPC scheme (cf. Fig 5).

In order to mitigate this problem, we first propose a simple patch which consists of accounting in the model used by either MPC scheme the prediction errors observed at the previous iterations. Essentially, this means that the controller imputes its prediction errors to model errors (i.e. those related to the fact that he does not account for the behavior of the neighboring areas, but also those that could be related to approximations 

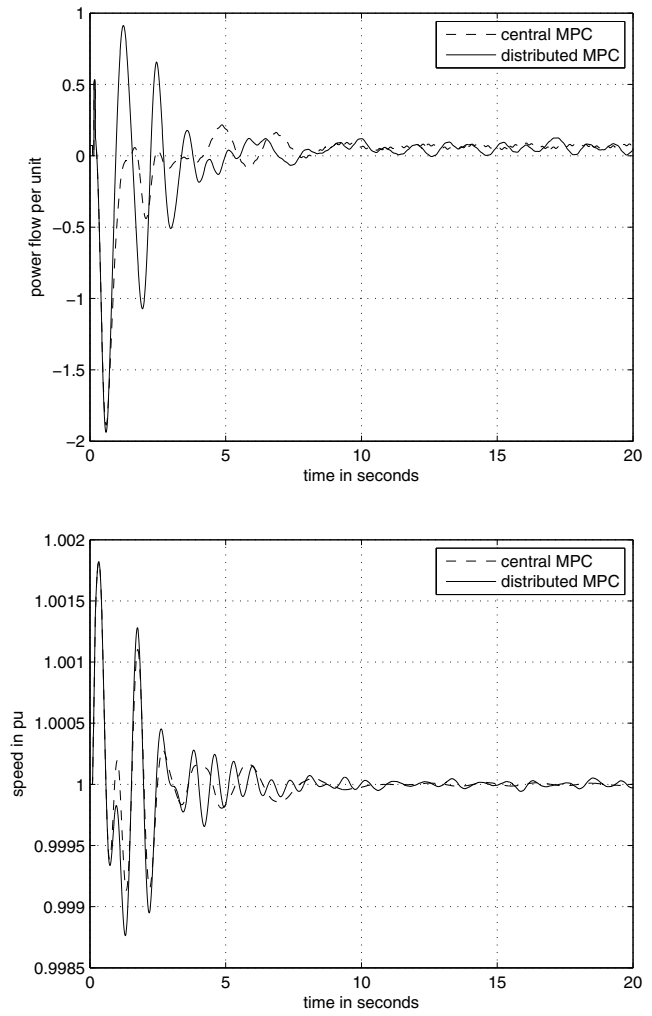

Fig. 6. Response of central MPC (dashed) and implicitly coordinated distributed MPC (plain): (top) active power of tie-line 1-2; (bottom) angular speed of generator 1

about his own control area, such as linearization, or model errors, or stochastic effects).

To this end, we used an additional output feedback term remaining unchanged over the entire prediction horizon, to be added to Eqn (4) so as to correct errors in the prediction of interactions with other areas. The new output equation of subproblem $n$ is:

$\left[\begin{array}{c}\hat{y}_{n}[1] \\ \hat{y}_{n}[2] \\ \vdots \\ \hat{y}_{n}[K]\end{array}\right]=P_{x . n} \hat{x}_{n}(t)+P_{u . n}\left[\begin{array}{c}u_{n}[0] \\ u_{n}[1] \\ \vdots \\ u_{n}[K-1]\end{array}\right]+\left[\begin{array}{c}y_{r . n}-y_{n}(t) \\ y_{r . n}-y_{n}(t) \\ \vdots \\ y_{r . n}-y_{n}(t)\end{array}\right]$

where $y_{r . n}-y_{n}(t)$ is the measured discrepancy between the reference and the observed performance of the local subsystem at the incept of the computation of optimal controls.

The use of this scheme is compared in Fig. 7 with the scheme of the previous section. We observe that the introduction of output feedbacks obviously improves control scheme performances, both in terms of angular speed of generator 1 and damping the power flow oscillations in line 1-2.

\section{Weakly coordinated MPC scheme II}

If active power increments of tie lines are damped as well as generator speed deviations, inter-area oscillations are damped completely. So, we introduce active power increments of tie
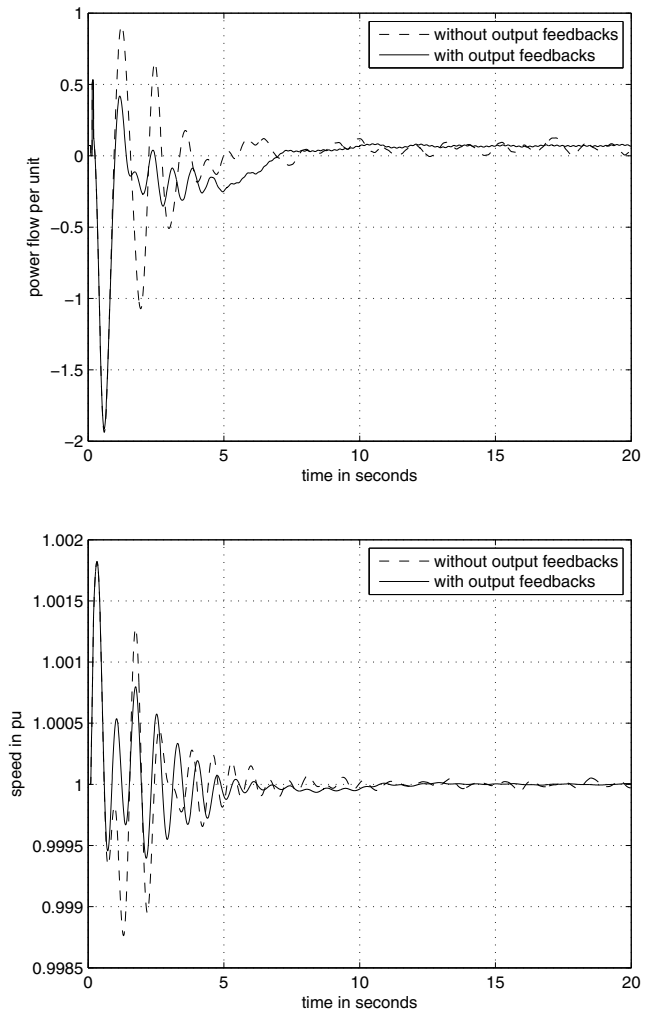

Fig. 7. Response of implicitly coordinated distributed MPC (dashed) vs weakly coordinated distributed MPC scheme I (plain): (top) active power of tie-line 1-2; (bottom) angular speed of generator 1

lines $y_{e x}$ within the objective function. Local MPC controllers thus not only drive their generators to run at base frequency, but also aim at making increments of exchange power with their neighbors reduce to zero. The new objective function of subproblem $n$ is rewritten as:

$$
\begin{array}{r}
\min _{u_{n}[\cdot]}\left\{\sum_{i=1}^{K}\left(\hat{y}_{n}[i]-y_{r . n}\right)^{T} Q\left(\hat{y}_{n}[i]-y_{r . n}\right)\right. \\
\left.+\hat{y}_{\text {ex.n }}[i]^{T} R \hat{y}_{\text {ex.n }}[i]\right\}
\end{array}
$$

System response using this formulation is shown by solid curves in Fig 8. The penalty item of exchange power increments strengthens coordination between local MPC controllers in order to damp inter-area oscillation more effectively. Small oscillations from $5 \mathrm{~s}$ to 20 s are indeed damped completely.

\section{CONClusion}

In this paper, we have considered distributed MPC to damp wide-area electromechanical oscillations in large-scale electric power systems and compared it with no coordinated control and with a fully centralized MPC based control.

Results obtained on a 16-generator, 70-bus, two-area test system show that distributed MPC schemes, even with no explicit coordination can effectively and quickly damp large inter-area oscillations in the first $5 \mathrm{~s}$, but with persistent small oscillations. Two simple improved distributed MPC schemes 

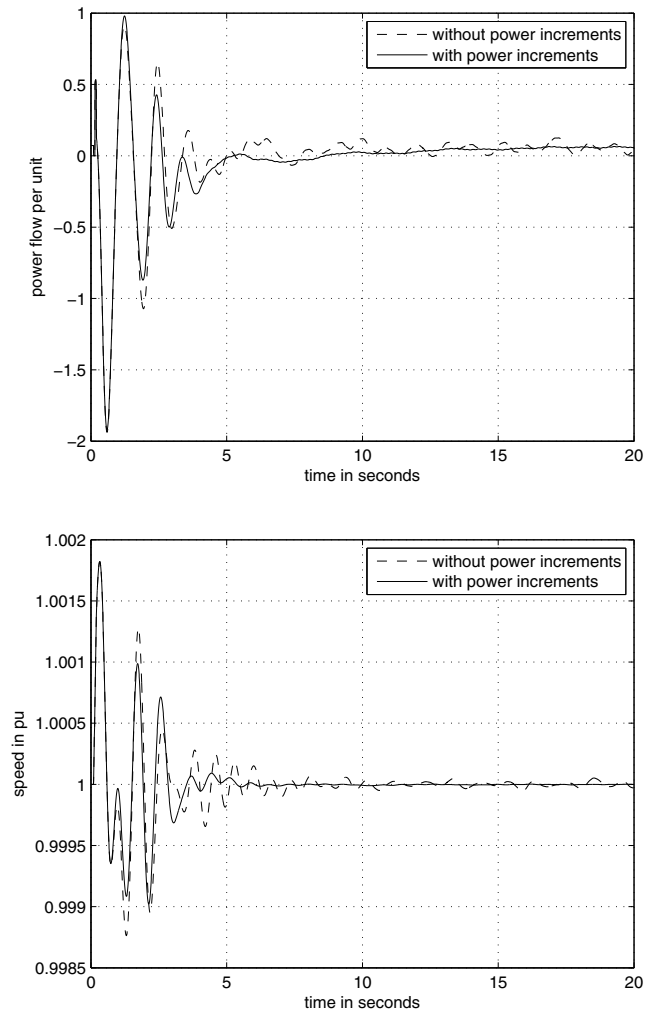

Fig. 8. Response of implicitly coordinated distributed MPC (dashed) and improved distributed MPC scheme II (plain): (top) active power of tie-line $1-2$; (bottom) angular speed of generator 1

were proposed with little overhead in terms of information exchange. Both have shown to be effective in solving the residual oscillation problem, and reached performances close to those of a fully centralized MPC scheme.

Further work should address ways to cope with overlapping models, measurement and control areas, and incorporate the practical constraints to further distribute control at the lower (intra-area) levels. Also, the theoretical investigation of stability guarantees of the proposed distributed MPC schemes is an important subject of further research.

While we do not believe at this stage that computational considerations are constraining in this problem, we believe that correct model construction is one aspect to keep in mind given the rapidly changing conditions and the need to better take into account the behavior of local subsystems (specially as concerns the distribution levels). Also, limitations in information exchanges due to lack of agreements or of technical communication channels may hinder progress. In these respects, automatic learning techniques could be useful to complement the model predictive control approach.

Eventually, one will be able to confront various distributed control approaches with various local objective functions, by leveraging modern information processing techniques.

\section{ACKNOWLEDGMENTS}

This paper presents research results of the Belgian Network
DYSCO, funded by the Interuniversity Attraction Poles Programme, initiated by the Belgian State and of the PASCAL Network of Excellence funded by the European Commission. The scientific responsibility rests with the authors.

\section{REFERENCES}

[1] G. Rogers, Power System Oscillations. Norwell, Massachusets, USA: Kluwer Academic Publishers, 2000.

[2] J. Lehner, T. Weissbach and G. Scheffknecht, "Oscillation behaviour of the enlarged UCTE power system including the Turkish power system". Proceedings of the 17th IFAC World Congress, Korea, July, pp. 94049409, 2008.

[3] E. Grebe, J. Kabouris, S. Lopez Barba, W. Sattinger and W. Winter, "Low frequency oscillations in the interconnected system of continental Europe". Proceedings of IEEE Power and Energy Society General Meeting 2010, Minneapolis, MN, July, 2010.

[4] D. Wang, M. Glavic and L. Wehenkel, "A new MPC scheme for damping wide-area electromechanical oscillations in power systems", Proceedings of the 2011 IEEE PES PowerTech, Trondheim, Norway, June, 2011.

[5] S. J. Qin and T. A. Badgwell, "A survey of industrial model predictive control technology", Control Engineering Practice, Vol. 11, pp. 733764, 2003.

[6] J. M. Maciejowski, Predictive Control with Constraints. Harlow, England: Prentice Hall, 2002.

[7] A. N. Venkat, Distributed Model Predictive Control: Theory and Applications. PhD thesis, University of Wisconsin-Madison, 2006.

[8] E. Camponogara, D. Jia, B. H. Krogh and S. Talukdar, "Distributed model predictive control", IEEE Control Systems Magazine, November, pp. 44-52, 2002.

[9] A. Monticelli, M.V.F. Pereira and S. Granville, "Security-constrained optimal power flow with post-contingency corrective rescheduling", IEEE Transactions on Power Systems, Vol. 2(1), pp. 175-180, 1987.

[10] B. Marinescu and H. Bourles, "Robust predictive control for the flexible coordinated secondary voltage control of large-scale power systems", IEEE Transactions on Power Systems, Vol. 14(4), pp. 1262-1268, 1999.

[11] B. Otomega, M. Glavic and T. Van Cutsem, "Emergency alleviation of thermal overloads using model predictive control", Proceedings 2007 IEEE Laussane PowerTech, July, pp. 201-206, 2007.

[12] S. Talukdar, D. Jia, P. Hines and B. H. Krogh. "Distributed model predictive control for the mitigation of cascading failurs", Proceedings of 44th IEEE Conference on Decision and Control, Seville, Spain, pp. 4440-4445, December, 2005.

[13] P. Hines, D. Jia and S. Talukdar. "Distributed model predictive control for electric grids", Proceedings of the Carnegie Mellon Transmission conference, Pittsburgh, USA, pp. 4440 - 2004, December, 2004.

[14] M. Larsson and D. Karlsson, "Coordinated system protection scheme against voltage collapse using heuristic search and predictive control", IEEE Transactions on Power Systems, vol. 18, no. 3, pp. 1115-1122, August, 2003.

[15] P. Li, B. H. Zhang, L. Y. Cheng, Z. Q. Bo and A. Klimek, "Study on the coordinated voltage control with the distributed model prediction", Proceedings of IEEE Power and Energy Society General Meeting 2010, Minneapolis, MN, pp. 1-6, July, 2010.

[16] A. G. Beccuti and M. Morai, "A distributed solution approach to centralized emergency voltage control", Proceedings of the 2006 American control conference, Minneapolis, MN, pp. 3445-3450, July, 2006.

[17] S. R. Wagh, A. K. Kamath and N. M. Singh, "Non-linear model predictive control for improving transient stability of power systems using TCSC controller", Proceedings of the 7th Asian Control Conference, Hong Kong, China, pp. 1627-1632, August, 2009

[18] L. Wang, H. Cheung, A. Hamlyn and R. Cheung, "Model predictive adaptive control of inter-area oscillations in multi-generator power systems", Proceedings of the IEEE PES General Meeting, Calgary, Canada, pp. 1-7, 2009.

[19] A. N. Venkat, I. A. Hiskens, J. B. Rawlings and S. J. Wright, "Distributed MPC strategies with application to power system automatic generation control", IEEE Transactions on Control Systems Technology, vol. 16, no. 6, pp. 1192-1206, November, 2008.

[20] R. R. Negenborn, Multi-agent model predictive control with applications to power networks, $\mathrm{PhD}$ thesis, Delft University of Technology, 2007. 
[21] N. Motee and B. S. Rodsari, "Optimal partitioning in distributed model predictive control", Proceedings of the 2003 American Control Conference, Colorado, USA, pp. 5300-5305, June, 2003. 